\title{
Retos contemporáneos para la formación permanente del profesorado universitario
}

\section{Carlos Andrés Benavides León}

https://orcid.org/o0oo-0003-3547-9835 Universidad Pontificia Bolivariana,

Colombia

carlos.benavidesl@upb.edu.co

\section{Nelly Milady López Rodríguez}

https://orcid.org/0000-0001-6064-990X Universidad Autónoma de Bucaramanga, Colombia nlopez413@unab.edu.co

\section{Resumen}

El artículo aborda la formación permanente del profesorado universitario, a partir de las tendencias y desarrollos conceptuales presentes en la bibliografia consultada. El objetivo del estudio es analizar los principales aportes a la formación del profesor universitario en la actualidad, con base en las dimensiones y competencias propuestas para el perfil del docente universitario y atendiendo a los desafios que presenta el contexto del siglo XXI a quienes ejercen la docencia universitaria o a quienes gestionan el desarrollo profesoral en las instituciones educativas.

\section{Palabras clave (Fuente: tesauro de la Unesco)}

Calidad de la educación; calidad de la enseñanza; competencias del docente; educación superior; enseñanza superior; evaluación de profesores; formación de docentes; formación de profesores.

Recepción: 30/10/2019 | Envío a pares: 05/03/2020 | Aceptación por pares: 07/03/2020 | Aprobación: 13/03/2020 


\title{
Contemporary Challenges to Ongoing Professor Training
}

\begin{abstract}
The article addresses the ongoing training of the faculty under the conceptual trends and developments found in the literature reviewed. The study aims to discuss chief contributions to professor training at present based on the dimensions and skills proposed for their profile. It also considers the challenges that the context of the 21st century poses to those involved in university teaching or managing teacher development in educational institutions.
\end{abstract}

\section{Keywords (Source: Unesco Thesaurus)}

Educational improvement; educational quality; teacher qualifications; higher education; university education; teacher assessment; teacher training; teacher education. 


\title{
Desafios contemporâneos para a formação permanente de docentes universitários
}

\begin{abstract}
Resumo
Este artigo aborda a formação permanente de docentes universitários a partir das tendências e desenvolvimento conceituais presentes na bibliografia consultada. $O$ objetivo deste estudo é analisar as principais contribuições para a formação do professor universitário na atualidade, com base nas dimensões e competências propostas para o perfil desse profissional e atendendo aos desafios que o contexto do século XXI formula a quem exerce a docência universitária ou a quem administra o desenvolvimento dos professores nas instituições educativas.
\end{abstract}

Palavras-chave (Fonte: tesauro da Unesco)

Qualidade da educação; qualidade de ensino; competências de professores; educação superior; ensino superior; avaliação de professores; formação de professores. 


\section{Introducción}

Los profesores universitarios representan el pilar sobre el cual se sustentan las funciones sustantivas de docencia, investigación y proyección social encomendadas a la educación superior por el Estado colombiano. Cada una de estas funciones constituye el fin último de la educación superior en Colombia de acuerdo con la Ley 30 de 1992 (Congreso de Colombia, 1992) y representan el horizonte que guía los procesos educativos en las universidades, instituciones universitarias e instituciones para la formación y el desarrollo humano. Para el logro de los fines de la educación superior es necesario contar no solamente con políticas y lineamientos, sino con profesores cuyo conocimiento disciplinar y pedagógico permita estructurar procesos de enseñanza que favorezcan el aprendizaje de los estudiantes, quienes constituyen la razón de ser del proceso educativo. Es allí donde emerge la pertinencia de la investigación sobre la formación permanente de los profesores universitarios, como modo de documentar, analizar, estructurar y diseñar procesos de formación permanente con los cuales atender a los desafíos de los procesos de la sociedad del conocimiento.

En ese contexto surgió el presente estudio, cuyo objetivo principal es investigar las aportaciones de la literatura especializada a la formación permanente de los profesores universitarios con el fin de construir un estado de la cuestión que dé cuenta de la formación permanente del profesorado universitario y permita responder de forma pertinente a los desafíos que impone el fortalecimiento del perfil de profesor universitario en todas sus dimensiones, mediante procesos de formación permanente. Los antecedentes encontrados en el ámbito regional, nacional e internacional permiten apreciar la importancia de esta temática para garantizar la materialización de las funciones sustantivas de la educación superior (Ley 30 de 1992), de modo que los hallazgos y conclusiones iluminen su desarrollo.
La pertinencia del estudio del desarrollo profesoral como una categoría válida encuentra sustento en la evolución del concepto de capacitación hacia el desarrollo. Ello implica procesos de metacognición sobre el quehacer docente, cuya existencia, desde la perspectiva de la capacitación, no puede ser identificada fácilmente, mientras que desde la perspectiva de desarrollo implica un carácter incremental de las competencias que se pretende alcanzar y, por tanto, implica la existencia de un proceso reflexivo que acompañe la formación del profesor, no solo por parte del profesor, sino desde las instituciones que diseñan y proporcionan los espacios de formación.

Otro asunto relacionado con la formación permanente del profesorado es el de las características de las sociedades industrializadas actuales: la globalización, los drásticos cambios tecnológicos, los cambios políticos y sociales y las reformas de las economías mundiales han reestructurado los sistemas sociales y han afectado, entre otras organizaciones, a las instituciones de educación superior, las cuales tienen como función formar profesionales a través de diversas metodologías y considerando múltiples áreas de conocimiento (Favoreto, Zago y Souza, 2017). Según Bermúdez-Aponte y Laspalas (2017), los cambios que se han sucedido en los ámbitos universitarios han afectado tanto el entorno como la propia concepción de la enseñanza, e inciden de manera diversa en la labor docente universitaria.

Desde la perspectiva de cambio, la formación del profesorado adquiere diversidad de dimensiones particulares que pueden encontrarse en la literatura disponible, en la que abundan las alusiones a la evolución del concepto de formación y desarrollo profesoral, a la capacitación y, últimamente, a la reconceptualización y resignificación de la labor docente. El presente trabajo de investigación parte de tres constructos principales: a) la formación del profesorado desde la perspectiva de contexto, b) la formación del profesorado como proceso permanente y c) la formación del profesorado como vehículo para significar la evaluación de desempeño. 


\section{Método}

En relación con el proceso metodológico, se ha considerado un enfoque metodológico cualitativo, dada la necesidad de obtener una comprensión de la formación permanente del profesorado universitario en lo relacionado tanto con la educación continua como con la posgradual, a partir de las aportaciones de los estudios disponibles sobre la formación de docentes en servicio a nivel superior, en los ámbitos regional, nacional e internacional. Hernández et al. afirman que "los métodos cualitativos representan un conjunto de procesos sistemáticos, empíricos y críticos de investigación e implican la recolección y el análisis de datos cualitativos, así como su integración y discusión, para realizar inferencias producto de toda la información recabada (metainferencias) y lograr un mayor entendimiento del fenómeno en estudio" (2014, p. 534).

En ese sentido, se analizaron cincuenta y dos (52) publicaciones, entre artículos, capítulos de libro y libros resultado de investigación, lo cual implicó consultar las bases de datos Academic Search Complete, Education Search Complete, Redalyc, Sciencedirect, Scielo y Dialnet. Para ello, se estableció una ventana de exploración de 10 años, contados a partir de 2008, y como idiomas de exploración se eligieron dos principalmente: inglés y español. Adicionalmente se incluyó un criterio de búsqueda relacionado con el lugar de desarrollo del estudio que sustentó el artículo, capítulo de libro o libro, lo cual permitió desvelar algunos de los sistemas educativos con mayores desarrollos investigativos relacionados con la formación permanente del profesorado.

El proceso metodológico incluyó tres fases principalmente: 1) la exploración propiamente dicha: consistió en la búsqueda, identificación y sistematización de los artículos, capítulos de libro o libros resultado de investigación que conformaron la base central del estudio: 2) la selección: consistió en caracterizar los documentos encontrados en la fase de exploración en un formato creado para tal fin, en el cual se analizó la naturaleza de los estudios que motivaron la publicación, la metodología, las conclusiones y principales aportaciones de la publicación. Este diseño permitió el registro documental para el análisis sintético de los documentos acopiados mediante la generación de tablas dinámicas de análisis; 3) el análisis: consistió en emplear un formato de Resumen Analítico Especializado (RAE) para extraer la información pertinente de contenido, metodología, conclusiones y comentarios del investigador. Los RAE fueron transferidos al software Atlas $\mathrm{Ti}$, en donde se crearon tres categorías apriorísticas para el análisis de la información y se permitió la codificación de patrones emergentes que eventualmente condujeron a la configuración de tres categorías emergentes.

Para el acopio de información de las fuentes primarias en el Resumen Analítico Especializado se atendieron las dimensiones de formación permanente del profesorado desde la perspectiva del contexto, la formación como proceso permanente y la formación como vehículo para resignificar la evaluación de desempeño de los profesores universitarios. El formato para la captura de la información como Resumen Analítico Especializado se estructura de acuerdo con los siguientes apartados: tipo de publicación, título, autor(es), datos de edición, palabras clave, descripción [contenido, metodología, diseño de investigación, conclusiones], comentarios del investigador, nombres y apellidos de quien elaboró el RAE, fecha de elaboración y datos de la fuente de consulta. El análisis documental con la técnica de análisis de contenido estructural' permitió a los investigadores la categorización, para posteriormente develar las tendencias conceptuales en relación con las tres perspectivas adoptadas, así como con las categorías emergentes del estudio.

$1 \quad$ El análisis de contenido estructural es una técnica destinada a precisar la manera como una obra se configura en su interioridad con el fin de abordar su organización. Desde la perspectiva de Barrera (2009), es una técnica descriptiva de nivel subyacente, usada entre otras en las ciencias de la educación, con el fin de describir un fenómeno y ubicarlo dentro de otro u otros. 


\section{Resultados}

A continuación se exponen los hallazgos obtenidos de la sistematización y caracterización de las 52 publicaciones que constituyeron las unidades comprensivas que integran este estudio de revisión. Para ubicar el origen de las unidades comprensivas, se identifica el país de realización del estudio explorado y se presenta la caracterización del origen del estudio (Figura 1). Esto permitió ubicar espacialmente las aproximaciones teóricas a la formación profesoral a nivel universitario, en las dimensiones que se presentarán más adelante.

Figura 1. Caracterización de las publicaciones por su origen

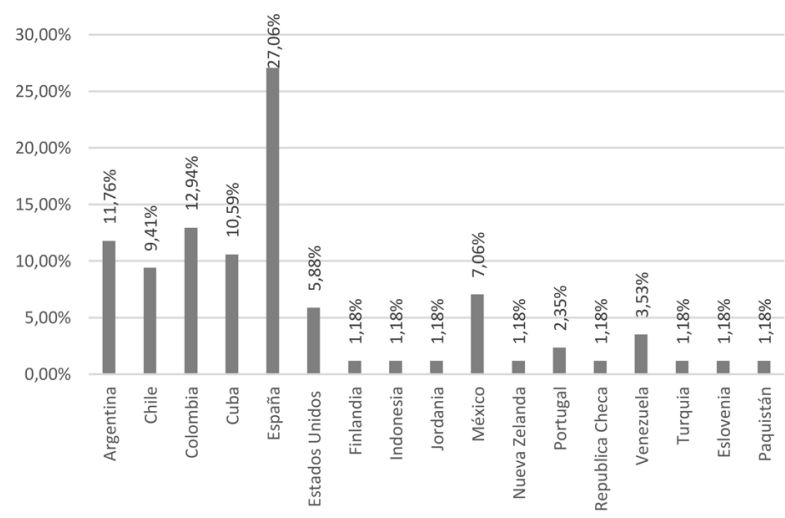

Fuente: elaboración propia.

Asimismo, el proceso de revisión bibliográfica permitió la ubicación temporal de la literatura explorada, la cual se puede encontrar en la Figura 2. Así fue posible encontrar una tendencia incremental en la abundancia de estudios sobre la temática en los últimos cuatro años, lo cual sugiere la ganancia de una mayor relevancia de la formación profesoral a nivel superior como un objeto de investigación entre la comunidad académica.

Los resultados se obtuvieron del análisis de contenido de las unidades comprensivas que conformaron la literatura revisada. Para ello se empleó el software Atlas TI a partir de tres categorías apriorísticas: formación con enfoque contextual, formación profesoral como proceso permanente y resignifica- ción de la evaluación docente. Asimismo, se permitió la emergencia de categorías no contempladas en las tres primeras. En la exploración se identificaron 81 unidades comprensivas potenciales distribuidas como se muestra en la Figura 3.

\section{Figura 2. Caracterización de las publicaciones por año de publicación}

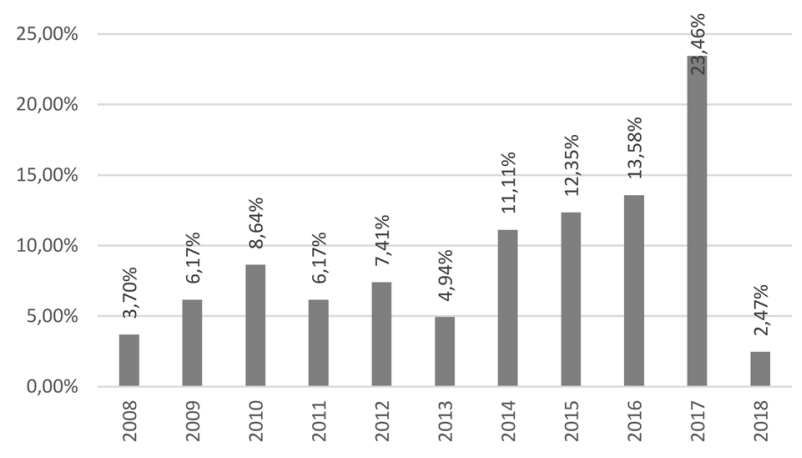

Fuente: elaboración propia.

Figura 3. Total de publicaciones seleccionadas en la fase de exploración

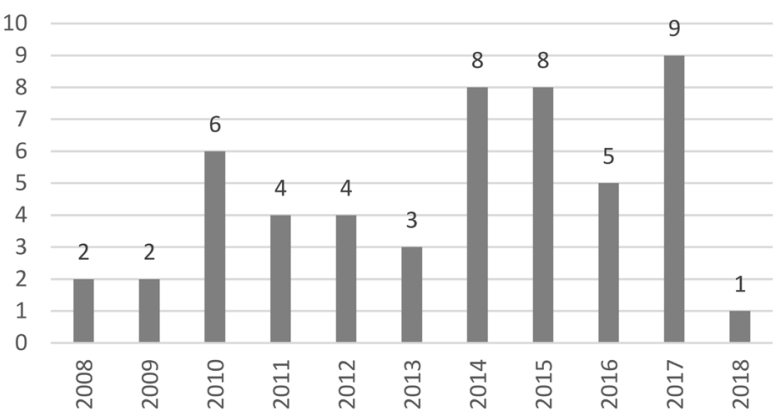

Fuente: elaboración propia.

A partir de este número de unidades comprensivas se realizó un análisis preliminar para determinar cuáles de estas unidades se tendrían en cuenta para la fase de análisis mediante el Resumen Analítico Especializado (RAE). Para ello se realizó una caracterización inicial de las unidades comprensivas, en la cual se tuvieron en cuenta los siguientes criterios:

1. Que las publicaciones fueran resultado de un proceso de investigación. 
2. Que su metodología fuera identificable.

3. Que su alcance fuera identificable en el cuerpo del texto.

4. Que se pudiera identificar el enfoque metodológico (cualitativo, cuantitativo o mixto).

5. Que aportaran en sus conclusiones a la comprensión del objeto de estudio: la formación permanente del profesorado universitario.

A partir de este análisis preliminar se pudieron aislar aquellos documentos que respondieron a los cinco criterios planteados anteriormente y se llegó a la cantidad de documentos analizados, como se observa en la Figura 4.

Figura 4. Total de publicaciones seleccionadas para el RAE

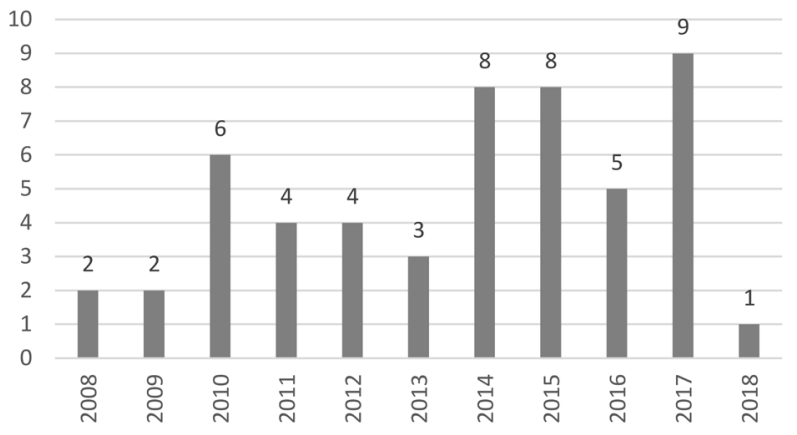

Fuente: elaboración propia.

Este grupo de 52 documentos fue el que se analizó mediante el RAE. En ese sentido, los resultados del análisis de contenido de las publicaciones seleccionadas, llevadas al Atlas Ti para su respectivo procesamiento, correspondieron con este número. En primer lugar, se realizó la codificación apriorística de los RAE en las tres grandes categorías: la formación desde una perspectiva contextual, la formación como proceso permanente y la formación como vehículo para dotar de significado los procesos de evaluación del desempeño docente. Un análisis preliminar en Atlas Ti permitió identificar los conceptos con mayor ocurrencia dentro del documento de RAE respecto de algunos de los aspectos asociados a la formación permanente del profesorado, tales como el desarrollo, la calidad, las competencias, el conocimiento, la tecnología, el aprendizaje, entre otros, como se puede apreciar en la nube de conceptos de la Figura 5.

Figura 5. Conceptos emergentes del análisis preliminar

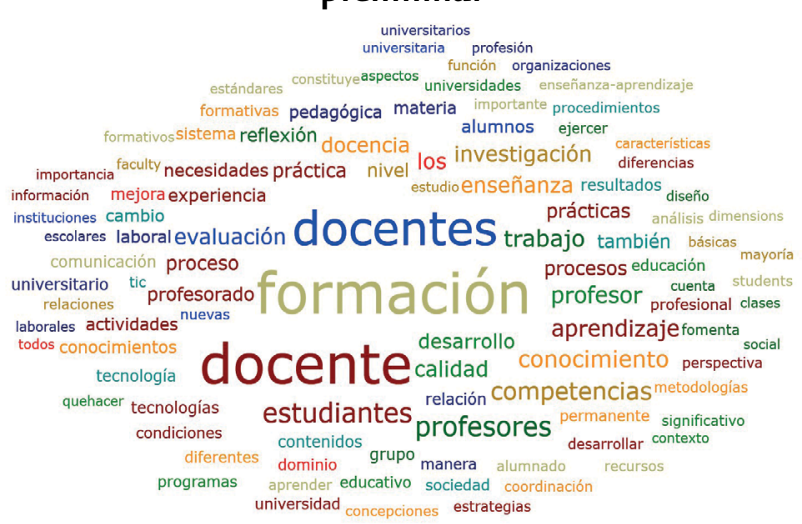

Fuente: elaboración propia.

Adicionalmente, se contempló la posibilidad de otras categorías emergentes en el análisis de los RAE. Para ello, se recurrió principalmente a un análisis detallado de la descripción, el contenido y las conclusiones de los estudios revisados, teniendo en cuenta que cada uno de los RAE presentaba esta estructura definida con antelación. En dicho análisis, realizado en el software Atlas $\mathrm{Ti}$, se pudo identificar una fuerte tendencia de la literatura a abordar las competencias requeridas para el ejercicio docente en el nivel superior y a estudiar los desafíos que presentan las condiciones del siglo XXI para el ejercicio de la docencia universitaria, aunado con la relevancia que tiene para el profesorado recibir formación permanente que le permita, además de perfeccionar conocimientos disciplinares y competencias para el ejercicio de la docencia, la asunción de una práctica metacognitiva, cuyo propósito es esencialmente el cultivo de competencias para autoagenciar el proceso de construcción del perfil docente. La Figura 6 muestra la distribución porcentual de los documentos estudiados en cada una de las categorías mencionadas. 


\section{Figura 6. Categorias aprioristicas y emergentes por frecuencia}

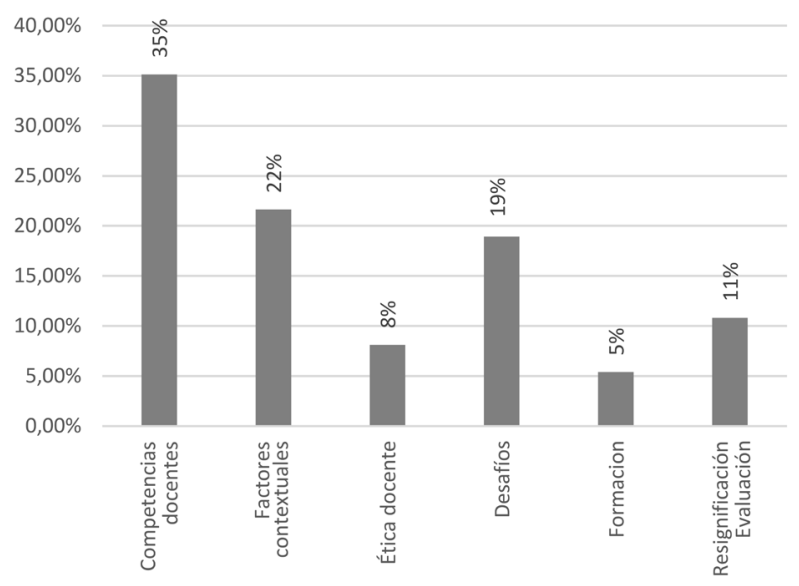

Fuente: elaboración propia.

Se puede observar que las competencias docentes son las que aparecen con mayor frecuencia en la literatura explorada en este estudio de revisión. Dichas competencias fueron: comunicación, didáctica, empatía, evaluación, disciplinares, idiomas, inclusión, innovación, inspiración, investigación, metacognición, uso de TIC.

Resulta relevante el hecho de que la literatura muestre una preocupación significativa por las competencias requeridas para el ejercicio docente, puesto que esto se convierte en un tipo de orientación tanto para los investigadores que asumen por objeto de estudio la formación profesoral como para las instituciones de educación superior que están llamadas a diseñar, construir y gestionar planes de formación permanente del profesorado, de manera que estos puedan responder de forma eficaz a los desafíos que presentan los aprendizajes en el contexto actual. Es de destacar que -además de las competencias tradicionalmente esperadas del profesorado, tales como la didáctica, la evaluación, los idiomas extranjeros, las TIC y la investigación- emergen otras, como la empatía, la comunicación, la inclusión (en un mundo sustentado en la diferencia), la metacognición y la inspiración, para convertirse en competencias relevantes para el ejercicio docente.
Hace tan solo unas décadas estas competencias que hoy se valoran en la literatura se asumían como meros rasgos o "formas de ser" del profesorado que no se vinculaban al adecuado ejercicio de la docencia. No obstante, hoy el contexto demanda profesores que sean capaces de innovar y reflexionar sobre lo que hacen, con una mirada crítica y sistémica, y que sepan comunicarse y situarse en el lugar del otro (empatía) e inspiren a sus educandos. Todas estas competencias emergen de demandas puntuales que el contexto hace al profesorado y que están sustentadas en retos particulares del siglo XXI relativos al ejercicio docente.

Precisamente los retos para el profesorado universitario son la segunda categoría con mayor frecuencia encontrada en la literatura explorada. Los retos están principalmente enfocados en la necesidad para el profesorado de estar equipado con las herramientas que le permitan responder de forma efectiva a los retos de la sociedad del conocimiento, en un contexto en el que, como en todos los sectores, se requiere del concurso de actores innovadores, capaces de reinventarse constantemente de acuerdo con los desafíos que se les presentan.

No obstante, también fue posible encontrar en la literatura explorada la creciente mercantilización de los procesos de enseñanza y aprendizaje, así como la asociación de las instituciones de educación a la estructura del mundo comercial y corporativo, mediante indicadores de productividad, eficiencia y sostenibilidad económica, cuya coherencia con el propósito inicial de la educación como un servicio y un derecho no siempre es posible conciliar. Así, en la categoría desafíos del siglo XXI se hallan como patrones los fenómenos de innovación, mercantilización y sociedad del conocimiento.

En relación con las categorías principales del inicio de la búsqueda, se pudo identificar patrones asociados a la formación permanente del profesorado mediante la conformación y construcción de comunidades de práctica que movilicen al profeso- 
rado a la coconstruccion y cocreación de experiencias de aprendizaje a partir de la interacción con pares de la propia y de otras instituciones de educación. Otro asunto que emergió de la revisión tiene que ver con el carácter continuo de la formación permanente del profesorado a través de experiencias formativas diversas.

La Tabla 1 presenta de forma resumida los hallazgos en la revisión en relación con las categorías mencionadas, en donde también se pueden apreciar los aspectos principales relativos a la naturaleza contextual que exigen los programas de formación del profesorado, en razón a la necesidad de dotarlos de pertinencia para satisfacer necesidades particulares de los entornos que se espera impactar con la formación de un determinado grupo de profesores, así como la necesidad de que existan estímulos e incentivos para el profesorado y que sus experiencias de formación cobren sentido. De este último aspecto, resalta en la revisión bibliográfica la imperiosa necesidad de estimular la profesión docente mediante su reconocimiento y la construcción de escenarios que permitan al profesorado volver sobre su práctica para evaluarla y repensarla a la luz de los desafíos que se le presentan.

Tabla 1. Categorias y patrones

\begin{tabular}{|c|c|}
\hline Categorías & Patrones \\
\hline $\begin{array}{c}\text { Formación } \\
\text { permanente }\end{array}$ & $\begin{array}{c}\text { Comunidades de práctica } \\
\text { Necesidades de formación continua }\end{array}$ \\
\hline $\begin{array}{c}\text { Factores } \\
\text { contextuales }\end{array}$ & $\begin{array}{c}\text { Contextualización } \\
\text { Formación disciplinar } \\
\text { Necesidades de formación } \\
\text { particulares } \\
\text { TIC }\end{array}$ \\
\hline $\begin{array}{c}\text { Resignificación } \\
\text { Evaluación } \\
\text { docente }\end{array}$ & $\begin{array}{c}\text { Estímulos e incentivos } \\
\text { Metacognición }\end{array}$ \\
\hline
\end{tabular}

Fuente: elaboración propia.

Finalmente, fue posible encontrar en la literatura un llamado a que los programas de formación permanente del profesorado incluyan una dimen- sión formativa en ética. Esto por sí mismo no resulta ser una novedad, si se tiene en cuenta que una de las funciones de cualquier profesor está asociada a la capacidad que tiene de "formar" a los estudiantes que le han sido confiados. No obstante, resulta revelador que la ética, como campo de estudio, se perciba como un componente transversal de formación para el profesorado al mismo nivel de las competencias que tanto la economía como los sistemas educativos plantean en el primer orden de los programas de formación.

\section{Discusión}

La formación del profesorado universitario desde la perspectiva de contexto emerge en la literatura como una necesidad imperiosa de dotar de competencias al profesor para aproximarse a una lectura de contexto que le permita situarse en las realidades que circundan su quehacer. Las competencias que el presente estudio permitió caracterizar aparecen mediadas por factores contextuales que permitan el ejercicio docente con pertinencia. Desde esta perspectiva contextual, en palabras de Pérez-Gómez, las instituciones de educación son "contextos epistémicos escolares que rodean el crecimiento de los aprendices y los repertorios de imágenes, creencias y artefactos que componen el conocimiento práctico del docente" (2010, p.171). Esto quiere decir que el conocimiento práctico del docente no solo está motivado por los grandes cambios que impactan globalmente la universidad como institución (tecnológicos, económicos, políticos, sociales y culturales), sino muy especialmente por las características contextuales y locales de su propio entorno institucional, que a su vez constituyen la columna vertebral para el desarrollo de los procesos de enseñanza-aprendizaje.

La pluridimensionalidad de los contextos demanda del profesor universitario no solamente una sólida formación disciplinar que le permita responder adecuadamente a las exigencias de conocimiento "de punta" que la sociedad y la economía 
requieren, sino también una formación pedagógica y metodológica con la cual estructurar procesos de enseñanza pertinentes para las características de los contextos en los que se pretende desarrollar la enseñanza, orientados a promover las competencias que los estudiantes demandan y vinculados a las características propias de un determinado contexto (Zabalza, Cid y Trillo, 2014). En esa misma línea, Izquierdo y Perdomo plantean la necesidad de adelantar procesos de formación del profesorado que respondan adecuadamente a las demandas que un determinado contexto va configurando en el proceso dinámico y natural de evolución, y añaden que la formación del profesor universitario es "un proceso educativo permanente [...] basado en la mejora profesional y apoyado en las necesidades prácticas de los profesores, que se desarrolla mediante la participación y que constituye un proceso de construcción profesional" (2014, p. 2). En ese sentido, no es posible abordar la formación del profesorado sin una adecuada delimitación y lectura del contexto en el cual y para el cual se requiere formar.

Las perspectivas que presenta la literatura contemporánea sobre la formación permanente del profesorado suponen una puesta en escena de mecanismos institucionales pertinentes que les permitan a los profesores universitarios desarrollar las competencias necesarias para construir un acervo que, a su vez, corresponda a la batería de herramientas esenciales para la planeación e implementación de la enseñanza en el contexto de la institución y en coherencia con sus fines particulares. De esta manera, la finalidad de la formación del profesorado está altamente influenciada por las características contextuales de los escenarios a los que está dirigida la práctica docente.

La formación del profesorado "no da lugar a discusión en ningún escenario, de orden institucional, nacional e internacional, por el contrario, se puede apreciar el consenso general al respecto, expresado en políticas públicas, esfuerzos institucionales para asegurar su desarrollo" (López, 2012, p. 53).
En ese mismo sentido, Zabalza et al. afirman que "la formación puede aportar a la construcción de la identidad docente, su función básica de mejora del aprendizaje de los estudiantes y sus aportaciones al desarrollo institucional" (2014, p. 45). En estos autores, se percibe el carácter contextual de la formación del profesorado, en tanto no solo su desarrollo está altamente ligado a los esfuerzos de las instituciones, sino que el impacto de dicha formación cobra sentido únicamente en los escenarios institucionales que pretende desarrollar.

Debido a que las instituciones de educación superior construyen su contexto a partir de las interacciones de distintos procesos sociales y económicos externos e internos, requieren aplicar cambios sustanciales en su estructura global con el fin de alcanzar una óptima adaptación a los cambios, generalmente complejos y emergentes (Vezub, 2009). Eso significa que la formación permanente del profesorado ya no obedece únicamente a una noción de capacitación, generalizada por décadas en diversos escenarios y fundamentada principalmente en el supuesto de que los profesores se "capacitan" para asumir un nuevo rol/perfil asignado mediante programas puntuales y enfocados en el desarrollo de algún tipo de habilidad técnica u operativa, sino que requiere de una construcción metodológica (Izquierdo y Perdomo, 2014) e identitaria (Keck y Saldivar, 2016) que contemple al estudiante como un "otro" que es al mismo tiempo centro y fin de la enseñanza (Krahenbuhl, 2016).

Esta tríada implica esencialmente que los procesos de formación del profesorado se sustenten en la necesidad de responder a contextos cada vez más complejos, una de cuyas características principales es el tránsito entre la relevancia asignada a la enseñanza y la asignada al aprendizaje, que pasa a ocupar un lugar protagónico. Adicionalmente, los contextos presentan mayor diversidad de interacciones entre los actores que participan, lo cual implica que el profesor universitario dota de sentido su quehacer mediante la resignificación y recontextualiza- 
ción de su labor, lo cual solo es posible cuando los procesos de formación permanente del profesorado se hacen de forma sistemática, planeada, consciente y propositiva. Valiente, Góngora, Torres y Otero abordan el papel del profesorado universitario desde la perspectiva de sistematicidad para garantizar "la integralidad de su formación, en función de un desempeño exitoso en los procesos sustantivos que concretan el compromiso social de dichas instituciones" (2013, p. 93).

Desde la perspectiva conceptual de acercamiento a la formación del profesorado se hace referencia a un tipo de relaciones más o menos precisas entre el fenómeno estudiado y otros aspectos de la vida humana y académica, pues ningún fenómeno se presenta en forma aislada, sino en relación con elementos históricos, sociopolíticos, económicos y culturales. Y si bien es pertinente estudiar las superficies locales de los diferentes procesos y problemáticas, lo cierto es que en casi todos los casos es necesario ampliar los rangos espacio-temporales de las problemáticas estudiadas con el fin de obtener una mayor inteligibilidad de ellos. Como plantea el pensador francés Edgar Morin (2000):

Los problemas esenciales nunca son parcelarios y los problemas globales son cada vez más esenciales. Además, los problemas particulares sólo pueden ser planteados y pensados correctamente dentro de su contexto, y el contexto mismo de estos problemas debe ser planteado cada vez más dentro del contexto planetario. (citado en Rodríguez, 2017, p. 426)

Con base en lo anterior, es pertinente decir que la lectura del contexto de la formación del profesorado en una determinada institución tiene que ver con establecer la jerarquía y necesidad de las relaciones que se tejen entre una problemática específica y los diferentes elementos que la componen sin confundirse con ella. Así, pues, el análisis del contexto implica reconocer el lugar del profesor en la cultura institucional y sus relaciones con las comunidades en las cuales interactúa.
De otro lado, la formación del profesorado, a la luz de la literatura explorada en este estudio, emerge como un proceso con carácter permanente, sistemático y metódico. Esto comporta la capacidad para dotar de oportunidades de recontextualización y reconstrucción de los saberes asociados al ejercicio de la docencia en la educación superior.

Según Bernstein (citado en Vezub, 2009), el perfeccionamiento docente surge como un espacio subordinado y con escasa autonomía, en relación tanto con el discurso pedagógico oficial como con el discurso regulador que crea los órdenes especializados y genera las identidades (Vezub, 2009). Por ende, se ha vuelto fundamental, para garantizar la sostenibilidad y sustentabilidad de las instituciones, que los procesos de formación del profesorado tengan un carácter permanente que les permita construir las herramientas necesarias para desenvolverse en contextos dinámicos e inciertos, como los que se les presentan. Para Sánchez, Lara, Bravo y Carballosa, "el desarrollo profesional es un proceso que se relaciona con la formación permanente del profesor universitario, caracterizado por la transformación de los conocimientos, habilidades y valores que contribuyen a la mejora de la calidad de la práctica educativa" (2014, p. 289), y añaden el carácter "planificado, permanente, continuo y sistémico de crecimiento que ubica la autopreparación como médula del trabajo metodológico" (p. 304).

Una de las características actuales es la rapidez y el dinamismo de los cambios. Esto obliga a que los procesos de formación del profesorado se transformen con el mismo ritmo y puedan responder adecuadamente a los retos que los cambios les presentan. Howard, McGee, Schwartz y Purcell (2000) plantean que la creación de ambientes de formación con un componente experiencial puede contribuir a lograr cambios más rápidos en las creencias y prácticas de los docentes, que eventualmente conduzcan a cambios significativos en los procesos de enseñanza. 
Ahora bien, la práctica, como lugar privilegiado y objeto de reflexión, significa, en muchos casos, que se debe hacer consciente y público lo que se hace bien, y en otros, que se tiene que plantear una transformación de uno mismo, de los contenidos, de las didácticas y de los objetivos de enseñanza. El profesorado está llamado a reflexionar acerca de su práctica desde dos orillas: una, el conocimiento cierto, amplio y en construcción sobre los objetos que enseña; dos, la capacidad de presentar esos contenidos de maneras no convencionales para que los estudiantes alcancen mejores aprendizajes (Rumayor, 2015).

Partiendo entonces del supuesto que la formación del profesorado es un proceso permanente que se construye en tanto exista la constante reflexión por parte del profesor, el primer paso es lograr que se haga dicha reflexión: ¿qué somos en tanto profesores universitarios? De tal manera: reflexión, conocimiento, acción y transformación es el objeto y el método de formación del profesorado para una educación cada vez más parecida a los ideales sociales que la promueven. Esta reflexión no solo contribuye a dotar de sentido y significado los procesos de formación del profesorado, sino que construye las características identitarias del YO profesor (Rumayor, 2015).

Para garantizar el carácter permanente de la formación del profesorado deben confluir aspectos académicos, administrativos e investigativos que alimenten los procesos de deconstrucción y reconstrucción, recontextualización del profesor universitario y su práctica. Sobre este aspecto, emerge la necesidad de pensar la gestión del talento humano, cuya función es la planificación, organización, dirección y control de los recursos, en relación con el personal al servicio de la empresa. Al respecto, Chiavenato señala que "los equipos de gestión del talento humano se libran de las actividades operativas y se ocupan de proporcionar asesoría interna para que el área asuma las actividades estratégicas de orientación global, de frente al futuro y al destino de la organización y de sus asociados" (2009, p. 42). Es decir, no pueden existir procesos de formación permanente si antes no existe un adecuado entorno administrativo que los posibilite.

Los equipos de gestión del talento humano, al identificar las necesidades y los profesores de la institución, pueden establecer los mecanismos de construcción, desarrollo, promoción y ejecución de programas que respondan con la debida pertinencia al carácter transformador y permanente que deben tener los procesos de cualificación de la práctica educativa del profesor universitario. Desde esta perspectiva, el equilibrio entre gestión académica, pedagógica y administrativa en las instituciones de educación superior juega un rol preponderante para generar la movilización de recursos de talento humano, administrativos y académicos que permitan la planeación y el desarrollo permanente de procesos de formación del profesorado con los mismos objetivos institucionales (Sánchez et al., 2014).

Una tercera perspectiva, con algún grado de notoriedad y comunalidad entre los autores explorados en el presente estudio, tiene que ver con la formación del profesorado como vehículo para significar los procesos de evaluación del desempeño docente, cuya existencia se aprecia en la inserción de la educación en el campo corporativo, lo que ha tenido como consecuencia que los profesores universitarios hayan ido adquiriendo connotaciones similares a las de los empleados corporativos en procesos tales como la evaluación del desempeño. En ese sentido, Jara y Díaz-López (2017) plantean la evaluación docente como piedra angular que posibilita la mejora de los procesos formulados en las políticas educativas. El objetivo es, entonces, que la evaluación posibilite identificar y reasignar sentido a la práctica docente a través de una propuesta sólida de formación del profesorado para el desarrollo profesoral continuo, y de los procesos de enseñanza y aprendizaje, que son el fin supremo de la práctica docente. 
La interacción entre la evaluación de desempeño y los programas de formación del profesorado no puede ni debe ser lineal. Tal como plantean Jara y Díaz-López (2017), cuando el proceso de evaluación es legítimo, debe conducir necesariamente al desarrollo profesional del profesorado. Al mismo tiempo, los programas de formación del profesorado transforman y cualifican la práctica docente, lo cual aporta una nueva dimensión a la evaluación de desempeño al ofrecer una nueva manera de actuar sobre la institución y la sociedad en la que vive el profesor (Rodríguez, 2017); es decir, debe existir una interacción horizontal y cíclica entre estos dos aspectos, de manera que el uno realimente al otro para evolucionar de forma conjunta.

\section{Figura 7. Relación entre programas de formación del profesorado y evaluación de desempeño}

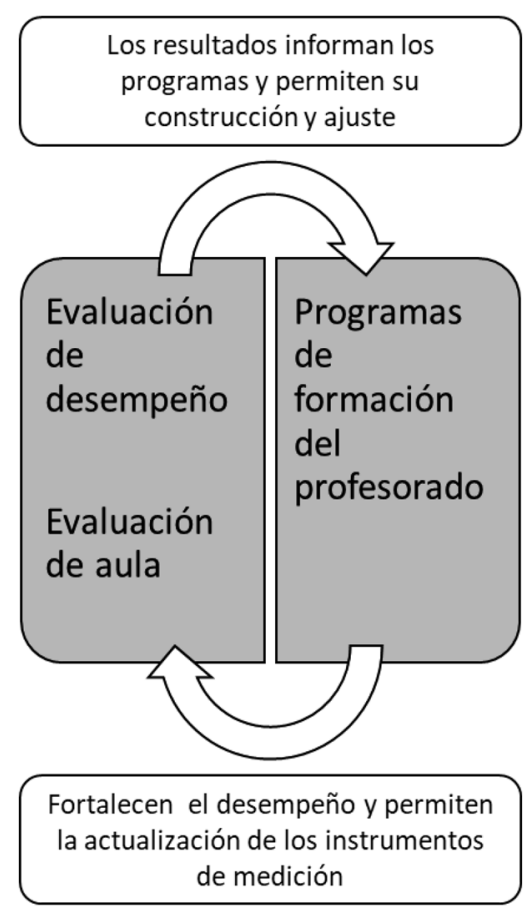

Fuente: elaboración propia.

Es importante explicar a qué tipo de evaluación nos referimos en el marco de este constructo. Al respecto, dice Tejada (citado en Cabero, Llorente y Morales, 2016) que la evaluación es una actividad procesual que persigue la emisión de un juicio de valor para adoptar decisiones, y no es una actividad meramente técnica, sino humana, por estar envuelta en valores éticos, estéticos y axiológicos. Es precisamente la confluencia de estos aspectos lo que hace que la evaluación abandone su intencionalidad inicial de calificación para convertirse en un ejercicio de valoración, lo que a su vez permite que tanto el profesor evaluado como la institución que lo evalúa establezcan una ruta de formación apropiada para el cumplimiento de las funciones de docencia, investigación y proyección social. Como afirman García, Tobón y López (2010, p. 90, citados en López, 2012) acerca de la evaluación de las competencias y del desempeño: "en definitiva, se trata de una evaluación valorativa que no solo permita a las directivas universitarias tomar decisiones sobre la permanencia de los profesores en la carrera docente, sino que informe pertinentemente las iniciativas de formación permanente del profesorado".

La interrelación atribuida entre procesos de formación del profesorado y evaluación de desempeño viene ganando un espacio en la discusión académica en torno a los aspectos que se espera que configuren el papel de la práctica docente. Sin embargo, no es fácil homogenizar los procesos de evaluación ni las interacciones que se establecen entre la evaluación y los programas de formación del profesorado, los cuales generan dinámicas propias que, en definitiva, configuran la relevancia o la falta de ella para el profesorado.

Para Farley, Clayton y Kaka, los profesores "necesitan entrenamiento específico sobre como reconciliar información inconsistente o contradictoria" $(2018$, p. 3). Esto quiere decir que el profesorado está constantemente sujeto a diversas formas de evaluar, con variaciones institucionales que implican que tanto los sistemas de evaluación como los programas de formación puedan dialogar con las características propias de una determinada cultura organizacional. Solo así cobrarán importancia en la cualificación de la práctica docente. 
Así pues, la formación del profesorado, vista como insumo y consecuencia de la evaluación docente, juega un doble papel. Por un lado, el de informar debidamente a las autoridades académicas sobre las necesidades y propósitos que debe suplir el proceso de formación permanente; por otro lado, el de resaltar la necesidad de que los procesos evaluativos sirvan al objetivo de generar verdaderas transformaciones en el personal docente y, por lo tanto, el de validar la pertinencia de un programa de formación del profesorado.

En resumen, la práctica docente es un continuum que requiere la revisión, actualización y contextualización de saberes y prácticas pedagógicas, a partir de elementos teóricos conceptuales y prácticos. Los planes de mejoramiento continuo que se desprenden de los ejercicios reflexivos deben responder a las demandas de las tendencias actuales sobre currículo y pedagogía y sobre evaluación de los aprendizajes y del desempeño docente, de modo que permitan abordar las necesidades, características y condiciones de la práctica docente.

Finalmente, la formación del profesorado universitario -desde una perspectiva de contexto, con carácter permanente y que les aporte valor a procesos como la evaluación del desempeño- constituye una apuesta por dotar de integralidad los procesos de formación e implica abandonar la noción de capacitación, cuyo alcance no permite la constante deconstrucción y reconstrucción de la práctica docente.

\section{Conclusión}

Hay evidencia de que los procesos tanto de formación continua como titulada a nivel de posgrado hacen parte de la cotidianidad del profesor universitario, lo cual pudo ser constatado en la abundancia de estudios de los últimos años sobre la temática. Lo anterior mostró un nivel significativo de adopción de una cultura del aprendizaje a lo largo de la vida.

Fue posible identificar elementos críticos en relación con la necesidad de plantear experiencias de formación profesoral novedosas y pertinentes que lleven al profesorado a aprovechar los espacios de formación a los cuales tienen acceso. De igual manera, se identificaron algunas aproximaciones teóricas, de las cuales destacó la asociación entre el ejercicio docente y el carácter dinámico de los contextos para los cuales se prepara el quehacer docente, independientemente del momento en que haya egresado de su proceso formativo.

Esto implica un doble desafío para futuros planes de formación docente: primero, la inclusión de iniciativas que motiven y convenzan al profesorado de la relevancia de estar inmersos en un proceso formativo permanente $y$, segundo, la necesidad de ir cambiando prejuicios acerca del ejercicio docente, mediante la visibilización de las "buenas prácticas" docentes que contribuyan a derrumbar la creencia generalizada sobre el ejercicio docente que la concibe como un proceso altamente tradicionalista que necesita ser cambiado por completo.

Asimismo, se pudo identificar algunas estrategias en relación con la implementación de la formación del profesorado, entre las que destaca la formación virtual y la vinculación de otras universidades o de entidades gubernamentales para cualificar el talento profesoral desde una perspectiva contextual. Con ello se podrá diseñar experiencias de formación más auténticas y cercanas a la realidad de los entornos hacia donde está dirigido el ejercicio docente.

Entretanto, en la revisión bibliográfica destacó, además de las áreas comúnmente asociadas al ejercicio docente, tales como la didáctica y la evaluación, la necesidad de formar en competencias relacionadas con la mediación comunicativa y la relación con los otros. Los resultados en esta área llevaron a determinar algunas necesidades de formación relacionadas con formar para atender a poblaciones con necesidades educativas especiales. Y vale la pena resaltar esta necesidad, porque abre un espacio para la materialización de las iniciativas de inclusión, además que evidencia progresos en la preocupación por el otro. 
En esa misma línea, aparecieron necesidades de formación en innovación, las cuales constituyen un eslabón de relacionamiento con lo propuesto por los teóricos de la educación (Zabalza, Cid y Trillo, 2014; Bozu e Imbernon, 2010; Concepción, Fernández y González, 2015, entre otros) y el Ministerio de Educación Nacional (2013).

Uno de los puntos más destacados de la identificación y caracterización de necesidades está relacionado con los desafíos que presenta la sociedad del conocimiento, cuyo en enfoque permite diagnosticar con precisión los aspectos relevantes para mejorar el perfil del profesorado y que, por ende, estos puedan ser orientados a adelantar acciones de formación concretas en áreas pertinentes. Este proceso contribuiría a la resignificación de la evaluación, la dotaría de mayor sentido y la haría útil para la articulación entre la evaluación del desempeño y la formación permanente.

A partir de la identificación de los asuntos que atañen actualmente a la formación del profesorado universitario, se pueden resaltar los siguientes aspectos:

- La necesidad de establecer procesos formativos pertinentes para el momento histórico, económico y científico que está viviendo el profesorado.
- La inclusión de competencias transversales, como las ligadas a necesidades educativas especiales, la mediación comunicativa y la gestión de proyectos, como vehículo para vincular la empresa privada y el sector estatal, etc.

- La inclusión de una ruta de formación para la construcción del proyecto ético de vida (Tobón, 2012) y de competencia para autogestionar el aprendizaje a lo largo de la vida.

Finalmente, es de destacar que se hace necesario continuar estudiando el desarrollo docente como constructo de investigación. Esto permitirá ir especializando los temas abordados, tales como la atención focalizada a necesidades particulares del profesorado en función de su experiencia, titulación y nivel formativo, para orientar más específicamente las necesidades de formación y las competencias a fortalecer de determinados segmentos de la población profesoral. Se hace necesario investigar para desarrollar un modelo de evaluación y medición del impacto de la formación permanente que contribuya a estimar el valor real de determinadas estrategias de formación y el impacto que tienen en el profesorado.

\section{Referencias}

Anderson, L. W. et al. (eds.) (2001). A taxonomy for learning, teaching, and assessing: A revision of Bloom's taxonomy of educational objectives. Boston, MA: Allyn \& Bacon-Pearson.

Aspin, D., Chapman, J., Hatton, M. y Sawano, Y. (2012). International Handbook of Lifelong Learning. Springer.

Basto, S. (2011). De las concepciones a las prácticas pedagógicas de un grupo de profesores universitarios. Magis. Revista Internacional de Investigación en Educación, 3(6), 393-412. https://revistas.javeriana.edu.co/index. php/MAGIS/article/view/3547

Bermúdez-Aponte, J.y Laspalas, F. (2017). El profesor universitario: Integración entre lo personal y lo profesional. Revista Interuniversitaria, 29(2), 109-126. https://doi.org/10.14201/teoredu292109126

Bozu, Z. e Imbernon Muñoz, F. (2010). La formación docente en momentos de cambios: ¿Qué nos dicen los profesores principiantes universitarios? Profesorado. Revista de Currículum y Formación de Profesorado, 20(3), 467-492. https://recyt.fecyt.es/index.php/profesorado/article/view/54607 
ISSN 0123-1294 | e-ISSN 2027-5358 | Educ.Educ. Vol. 23. No.1 | Febrero-abril de 2020 | pp. 71-88.

Universidad de La Sabana | Facultad de Educación

Cabero Almenara, J., Llorente Cejudo, M. C. y Morales Lozano, J. A. (2018). Evaluación del desempeño docente en la formación virtual: ideas para la configuración de un modelo. Revista Iberoamericana de Educación a Distancia, 21(1), pp. 261-279. https://doi.org/10.5944/ried.21.1.17206

Cisterna, F. (2005). Categorización y triangulación como procesos de validación del conocimiento en investigación cualitativa. Theoria. Ciencia, Arte y Humanidades, 14(1), 61-71. http://www.ubiobio.cl/theoria/v/v14/a6.pdf

Concepción, M., Fernández, M. y González, D. (2015). Estudio descriptivo de las necesidades de formación docente del profesorado universitario novel. Pedagogía Universitaria, 2O(2). http://cvi.mes.edu.cu/peduniv/ index.php/peduniv/article/view/678

Congreso de Colombia (1992). Ley 30 de 1992. https://www.cna.gov.co/1741/articles-186370_ley_3092.pdf

Díaz Larenas, C., Solar Rodríguez, M., Soto Hernández, V.y Conejeros del Solar, M. (2015). Formación docente en Chile: percepciones de profesores del sistema escolar y docentes universitarios. Civilizar. Ciencias Sociales y Humanas, 15(28), 229-245. https://doi.org/10.22518/16578953.283

Espinosa, M. (2014). Necesidades formativas del docente universitario. Revista de Docencia Universitaria, 12(4), 161-177. https://doi.org/10.4995/redu.2014.5619

Farley, A. N., Clayton, G. y Kaka, S. J. (2018). Linking teacher education to redesigned systems of accountability: A call for multiple measures in pre-service teacher effectiveness. Education Policy Analysis Archives, 26(7-16), 1-11. https://doi.org/10.14507/epaa.26.3441

Favoreto, A., Zago Figueiredo, I. M. y Souza Zanardini, I. M. (2017). Formação docente: relação entre alienação e práxis reflexiva. Revista Práxis Educativa, 12(3), 980-994. https://doi.org/10.5212/PraxEduc.v.12i3.016

García-Retamero, R. y Hoffrage, U. (2009). Influencia de las creencias causales en los procesos de toma de decisiones. Revista Mexicana de Psicología, 26(1), 103-111. http://comeppsi.com/images/rmp/RMP\%20261\%20103-111.pdf

Hernández, R., Fernández, C. y Baptista, MdP. (2014). Metodología de la investigación. 6 ed. México: McGraw-Hill Interamericana.

Howard, B., McGee, S., Schwartz, N. y Purcell, S. (2000). The experience of constructivism: Transforming teacher epistemology. Journal of Research on Computing in Education, 32(4), 455-465. https://doi.org/10.1080/088 86504.2000.10782291

Izquierdo, Z. y Perdomo, J. (2014). La preparación metodológica del profesor universitario para la estimulación de la motivación por el estudio independiente. Revista Infociencia, 18(1), 1-12. http://www.infocienciass.cu/ index.php/infociencia/article/view/288

Jara, N.y Díaz-López, M. (2017). Políticas de evaluación del desempeño del docente universitario, mito o realidad. Revista Cubana de Educación Médica Superior, 31(2), 1-15. http://www.ems.sld.cu/index.php/ems/article/ view/1010 
Keck, C. S. y Saldívar Moreno, A. (2016). Una mirada a la formación docente desde 'la experiencia': Una apuesta por el no-futuro de la educación. Sinéctica, 47, 1-19. https://sinectica.iteso.mx/index.php/SINECTICA/ article/view/638

Krahenbuhl, K. S. (2016). Student-centered education and constructivism: Challenges, concerns, and clarity for teachers. The Clearing House: A Journal of Educational Strategies, Issues and Ideas, 89(3), 97-105. https://doi. org/10.1080/00098655.2016.1191311

López, B.y Basto, S. (2010). Desde las teorías implícitas a la docencia como práctica reflexiva. Educación y Educadores, 13(2), 275-291. https://doi.org/10.5294/edu.2010.13.2.6

López, R. N. (2012). Una mirada al desarrollo docente y la formación del profesorado en la Universidad Santo Tomás, Bucaramanga. Revista de Docencia e Investigación, 2(2), 37-56. https://doi.org/10.15332/erdi.v2i2.145

Macera, I. (2012). Un estudio de las concepciones docentes acerca de la formación permanente. Educación y Educadores, 15(3), 513-531. https://doi.org/10.5294/edu.2012.15.3.10

Martín Sospedra, D. R.y Herrera Rodríguez, J. I. (2014). La formación de los docentes universitarios para potenciar el aprendizaje desarrollador. Gaceta Médica Espirituana, 16(2), 118-126. http://revgmespirituana.sld.cu/index.php/gme/article/view/658/513

Medina, A., Domínguez, C. y Ribeiro, F. (2011). Formación del profesorado universitario en las competencias docentes. Revista Historia de la Educación Latinoamericana, 13(17), 119-138. https://revistas.uptc.edu.co/index.php/historia_educacion_latinamerican/article/view/160o

Ministerio de Educación Nacional (2013). Lineamientos para la acreditación de programas de pregrado. Bogotá: MEN.

Montes, D. y Suárez, C. (2016). La formación docente universitaria: claves formativas de universidades españolas. Revista Electrónica de Investigación Educativa, 18(3), 51-64. http://www.scielo.org.mx/scielo. php?script=sci_arttext\&pid=S1607-40412016000300004\&lng=es\&tlng=es

Morante, J. R. y Gómez, A. L. (2007). ¿Sirven las políticas y prácticas de formación del profesorado para mejorar la educación? Una respuesta desde el análisis de la construcción social de la docencia. Education Policy Analysis Archives, 15(19), 1-22. https://doi.org/10.14507/epaa.v15n19.2007

Moscoso Merchán, F. y Hernández Díaz, A. (2015). La formación pedagógica del docente universitario: un reto del mundo contemporáneo. Revista Cubana de Educación Superior, 34(3), 140-154. http://www.rces.uh.cu/ index.php/RCES/article/view/92

Parra-Moreno, C., Ecima-Sánchez, I., Gómez-Becerra, M. y Almenárez-Moreno, F. (2010). La formación de los profesores universitarios: una asignatura pendiente de la universidad colombiana. Educación y Educadores, 13(3), 421-452. https://doi.org/10.5294/edu.2010.13.3.6

Pérez-Gómez, Á. (2010). La naturaleza del conocimiento práctico y sus implicaciones en la formación de docentes. Infancia y Aprendizaje, 33(2), 171-177. https://doi.org/10.1174/021037010791114652 
Pichs, B., Sánchez, R., Hernández, D. y Benítez, F. (2006). La formación y desarrollo de los profesores en las sedes universitarias. La preparación psicopedagógica del tutor. Pedagogía Universitaria, 11(2), 44-53. http://cvi. mes.edu.cu/peduniv/index.php/peduniv/article/view/364/

Ramírez, D. (2006). La importancia de la evaluación del desempeño en la gerencia de la ciencia. Ciencias Holguin, 12(4), 1-8. http://www.ciencias.holguin.cu/index.php/cienciasholguin/article/view/362

Rodríguez, M. E. (2017). Currículum, educación y cultura en la formación docente del siglo XXI desde la complejidad. Educación y Humanismo, 19(33), 424-439. https://doi.org/10.17081/eduhum.19.33.2654

Rumayor, M. (2015). El raciovitalismo de Ortega y la universidad que buscamos. Revista Española de Pedagogía, 73(260), 73-87. https://revistadepedagogia.org/wp-content/uploads/2015/01/raciovitalismo-de-Ortega.pdf

Sánchez, L., Lara, L., Bravo, G. y Caballosa, A. (2014). Contribución del trabajo metodológico al desarrollo profesional del profesor universitario: un análisis desde la autopreparación. Ciencia y Sociedad, 39(2), 287-309. https://doi.org/10.22206/cys.2014.v39i2.pp287-309

Seijas, E., Muñoz, M. y Antelo, B. (2014). Las necesidades formativas del profesorado universitario: un análisis desde el programa de formación docente de la Universidad de Santiago de Compostela. Revista de Docencia Universitaria, 12(4), 203-229. https://doi.org/10.4995/redu.2014.5621

Tobón, S. (2006). Formación basada en competencias: pensamiento complejo, diseño curriculary didáctica. 2 ed. Bogotá: Ecoe.

Tobón, S. (2012). El proyecto ético de vida y la socioformación. Cuernavaca: Instituto CIFE. https://issuu.com/cife/ docs/e_book_el_proyecto_etico_de_vida_y_

Unesco (2002). Formación docente: reflexiones, debates, desafíos e innovaciones. Perspectivas. Revista Trimestral de Educación Comparada, 123(3), 265-271. https://unesdoc.unesco.org/ark:/48223/pfooo0129475_spa

Valiente, P., Góngora, G., Torres, J. y Otero, Y. (2013). La experiencia cubana en la formación del profesor universitario. Revista de Docencia Universitaria, 11(3), 91-123. https://doi.org/10.4995/redu.2013.5522

Vezub, L. F. (2009). Notas para pensar una genealogía de la formación permanente del profesorado en la Argentina. Revista Mexicana de Investigación Educativa, 14(42), 911-937. http://www.scielo.org.mx/scielo. php?script=sci_arttext\&pid=S1405-66662009000300014

Wilches, J., Amézquita, L.y Guerrero, H. (2016). De los viejos individualismos a las nuevas sociedades: tecnologías de la información en la cualificación de prácticas docentes universitarias. Revista Lasallista de Investigación, 13(1), 105-115. https://doi.org/10.22507/rli.v13n1a9

Zabalza, M., Cid, A. y Trillo, F. (2014). Formación docente del profesorado universitario. El difícil tránsito a los enfoques institucionales. Revista Española de Pedagogía, 72(257), 39-54. https://revistadepedagogia.org/ 1xxii/no-257/formacion-docente-del-profesorado-universitario-el-dificil-transito-a-los-enfoques-institucionales/101400010366/ 